\title{
Prediction of progression to muscle-invasive disease in patients with high-risk bladder cancer
}

\author{
Maciej Oszczudlowski, Jakub Dobruch \\ Centre of Postgraduate Medical Education, Warsaw, Poland \\ Correspondence to: Jakub Dobruch. Centre of Postgraduate Medical Education, Warsaw, Poland. Email: kubadobr@wp.pl. \\ Provenance: This is a Guest Editorial commissioned by Section Editor Xiao Li (Department of Urology, Jiangsu Cancer Hospital \& Jiangsu Institute \\ of Cancer Research \& Nanjing Medical University Affiliated Cancer Hospital, Nanjing, China). \\ Comment on: D'Andrea D, Abufaraj M, Susani M, et al. Accurate prediction of progression to muscle-invasive disease in patients with pT1G3 bladder \\ cancer: A clinical decision-making tool. Urol Oncol 2018;36:239.e1-239.e7.
}

Submitted Jun 04, 2018. Accepted for publication Jun 20, 2018.

doi: $10.21037 /$ tau.2018.06.14

View this article at: http://dx.doi.org/10.21037/tau.2018.06.14

Despite complete transurethral resection of bladder tumor (TURBT) and adjuvant intravesical treatment, relevant proportion of patients with non-muscle invasive bladder cancer (NMIBC) experience disease recurrence or progression (1). Furthermore, from those who experience progression to muscle invasive bladder cancer (MIBC), two thirds succumb to the disease within 48 months even after radical treatment (2). It has also been shown that cancer specific survival (CSS) is significantly worse when secondary MIBC has occurred, when related to primary muscle invasive disease. In such cases, upfront (early or immediate) radical cystectomy (RC) is preferred option, likely improving oncological outcomes and should be considered in a group of NMIBC patients with very high risk of progression (3). On the other hand, there are still a major proportion of patients who may be cured by conservative therapy and for whom RC is deemed as overtreatment. This highlights the need for accurate prediction of progression in patients with high risk NMIBC.

The most commonly used tool to this end is EORTC risk calculator. It is based on standard clinical and pathological features such as size and number of tumors, recurrence rate, tumor grade, clinical stage and the presence of concomitant carcinoma in situ (CIS). Combination of these features allows for assessment of the probability of cancer recurrence and progression. The calculator has been derived primarily from analysis of 2,596 patients with T1G3 tumors comprising around $10 \%$ of cases (4). As such, it is reliable to underpin those who would not progress or die of bladder cancer rather than those who would be diagnosed with MIBC. Furthermore, lack of consistent repeat transurethral resection (reTURBT) and bacillus CalmetteGuérin (BCG) maintenance therapy in selected patients additionally tempers its' current clinical application (3). Therefore, other prognostic measures of BC are broadly investigated. More recently published studies included patients treated with maintenance BCG for 1 to 3 years, however none of them had reTURBT, what could have contributed to understaging and hence, overestimating the risk of progression by proposed scoring system, especially in patients with T1G3 tumors (1). According to other data from meta-analysis of seven retrospective studies, combining the outcomes of 1,206 patients with NMIBC, lymphovascular invasion (LVI) in TURBT specimen, defined as tumor cells within or attached to the wall of a vascular or lymphatic space, was recognized as a significant risk factor for progression (pooled HR $=2.28,95 \% \mathrm{CI}$ : 1.45-3.58) (5). Additionally, several histologic variants and divergent differentiations of urothelial carcinoma, with different influence on prognosis, have been defined (6). Although, micropapillary, sarcomatoid or plasmacytoid variants are correlated with poor outcomes, differences in prognosis, compared to pure urothelial cell carcinoma, may not be so apparent in case of nested or small cell variants when corrected for stage of the disease (7). Especially, when taking into consideration high rate of understaging of the primary tumors with variant histology (VH) (8). Furthermore, despite being independently associated by 
most authors with worse outcomes, LVI and VH have not been incorporated to prognostic models yet.

D Andrea $e t$ al. have recently published results of their retrospective analysis of 1,289 patients with T1G3 bladder cancer who were treated with TURBT and BCG (9). Authors aimed to develop a clinical decision-making tool that integrated VH and LVI for selecting patients with T1G3 bladder cancer who would be at increased risk of disease progression. Prognostic model, based on 5 clinicopathologic features (LVI, VH, concomitant CIS, tumor multifocality and size), was developed and tested on equally sized derivation and validation cohorts respectively. Within a median follow-up of 56.6 months, from 1,289 patients, $553(42.4 \%)$ experienced disease recurrence while progression was observed in $166(12.7 \%)$ of them. LVI and $\mathrm{VH}$ were found in 115 (8.9\%) and 157 (12.2\%) patients respectively, while both features were identified in 34 (2.6\%) cases. In multivariate analysis LVI and VH were found to be associated with the highest risk of progression ( $\mathrm{HR}=3.5$ and 4.4 for LVI and VH respectively) being the two most predictive factors in the presented model. Based on the outcomes of 645 patients in the derivation cohort, estimated 5 -year risk of progression mounted to $38.7 \%, 47.3 \%$ and $78.3 \%$ for patients with LVI, VH and both features respectively compared to $7 \%$ for those with none of the above factors. Interestingly, neither tumor multifocality nor tumor size $>3 \mathrm{~cm}$ or concomitant CIS were found in multivariable competing-risk analysis to be significantly correlated with the risk of progression. These findings can be partially explained by the fact that this cohort represents, unlike other cohorts, high-risk population with relatively high progression rate (9). Moreover, relatively low prevalence of concomitant CIS (6.2\%) observed by authors may have influenced its prognostic value.

In practical considerations, nomogram derived from the mentioned analysis has been proposed to better identify patients with NMIBC who would benefit from early radical treatment. Assuming 20\% threshold of progression risk as the trigger for $\mathrm{RC}$, one may reduce the rate of unnecessary surgery by $64.8 \%$ in patients that would not experience disease progression, without missing a patient that would experience disease progression (9). However, several limitations that may compromise the accuracy of this nomogram should be acknowledged. First of all, despite reTURBT was declared as a policy at all of included centers (9), data regarding the rate of reTURBT and adherence to EAU indication for reTURBT was not provided. Hence, discrepancy between different centers regarding quality of treatment could not be ruled out. The same consideration can be made on the BCG treatment. Furthermore, lack of precise data about subtypes of $\mathrm{VH}$, in the context of its heterogeneity and different prognosis, may contribute to compromise its predictive value in particular patient.

Efforts to create precise decision-making tool for better identification of T1G3 patients who will progress and hence need upfront RC are consistent with the movement towards risk-based counseling and personalized medicine. Clinical and histological features, as mentioned above, are only some of investigated variables. In recent years, several promising molecular markers have been identified, however further validation is needed. Based on the genomewide analyses, molecular subtypes within two major groups (NMIBC and MIBC) were defined, providing potentially prognostic information. One of the first studies in this field defined five subtypes among tumors of all stages and grades (10). More recently, large multi-centre study on a group of 460 with NMIBC, has provided evidence for three expression classes. Class 1 contained many Ta tumours and showed best prognosis while class 2 and 3 contained more T1 high-grade tumours and those with a high EORTC risk score. These classes included the majority of patients who progressed to MIBC (11). Another promising marker of clinical importance is FGFR3 mutation linked to favourable prognosis in T1 patients (12).

In summary, D Andrea et al. demonstrated that LVI and $\mathrm{VH}$ are important histological features of predictive value. This statement is consistent with EAU guidelines including LVI and VH into definition of the highest risk tumors. Moreover, authors proposed nomogram, based on the cohort of high-risk patients treated with reTURBT and maintenance BCG, with better than EORTC and CUETO calculator discrimination in predicting progression of T1G3 BC. Due to the movement towards tailored-made treatment further molecular markers of NMIBC progression and validation of already existing ones in immediate future are expected.

\section{Acknowledgements}

None.

\section{Footnote}

Conflicts of Interest: The authors have no conflicts of interest to declare. 


\section{References}

1. Cambier S, Sylvester RJ, Collette L, et al. EORTC Nomograms and Risk Groups for Predicting Recurrence, Progression, and Disease-specific and Overall Survival in Non-Muscle-invasive Stage Ta-T1 Urothelial Bladder Cancer Patients Treated with 1-3 Years of Maintenance Bacillus Calmette-Guerin. Eur Urol 2016;69:60-9.

2. van den Bosch S, Witjes JA. Long-term Cancer-specific Survival in Patients with High-risk, Non-muscle-invasive Bladder Cancer and Tumour Progression: A Systematic Review. Eur Urol 2011;60:493-500.

3. Babjuk M, Bohle A, Burger $M$, et al. EAU Guidelines on Non-Muscle-invasive Urothelial Carcinoma of the Bladder: Update 2016. Eur Urol 2017;71:447-61.

4. Sylvester RJ, van der Meijden APM, Oosterlinck W, et al. Predicting recurrence and progression in individual patients with stage Ta T1 bladder cancer using EORTC risk tables: A combined analysis of 2596 patients from seven EORTC trials. Eur Urol 2006;49:466-77.

5. Kim HS, Kim M, Jeong CW, et al. Presence of lymphovascular invasion in urothelial bladder cancer specimens after transurethral resections correlates with risk of upstaging and survival: A systematic review and

Cite this article as: Oszczudlowski M, Dobruch J. Prediction of progression to muscle-invasive disease in patients with highrisk bladder cancer. Transl Androl Urol 2018;7(4):749-751. doi: 10.21037/tau.2018.06.14 meta-analysis. Urol Oncol 2014;32:1191-9.

6. Humphrey PA, Moch H, Cubilla AL, et al. The 2016 WHO Classification of Tumours of the Urinary System and Male Genital Organs-Part B: Prostate and Bladder Tumours. Eur Urol 2016;70:106-19.

7. Amin MB, McKenney JK, Paner GP, et al. ICUD-EAU International Consultation on Bladder Cancer 2012: Pathology. Eur Urol 2013;63:16-35.

8. Willis DL, Fernandez MI, Dickstein RJ, et al. Clinical Outcomes of c'T1 Micropapillary Bladder Cancer. J Urol 2015;193:1129-34.

9. D'Andrea D, Abufaraj M, Susani M, et al. Accurate prediction of progression to muscle-invasive disease in patients with pT1G3 bladder cancer: A clinical decisionmaking tool. Urol Oncol 2018;36:239.e1-239.e7.

10. Sjodahl G, Lauss M, Lovgren K, et al. A Molecular Taxonomy for Urothelial Carcinoma. Clin Cancer Res 2012;18:3377-86.

11. Hedegaard J, Lamy P, Nordentoft I, et al. Comprehensive Transcriptional Analysis of Early-Stage Urothelial Carcinoma. Cancer Cell 2016;30:27-42.

12. van Rhijn BWG, van der Kwast TH, Liu LY, et al. The FGFR3 Mutation is Related to Favorable pT1 Bladder Cancer. J Urol 2012;187:310-4. 